Ann. Génét. Sél. anim., I969, 1 (I), 33-37.

\title{
DÉTERMINISME HÉRÉDITAIRE DU CARACTERE « GRÊTE PÂLE » CHEZ LA POULE
}

\author{
F.-H. RICARD \\ avec la collaboration technique de Renée Bourdiou et J.-C. CAritez \\ Station expérimentale d'Aviculture du Magneraud, i 7 -Saint-Pierre-d'Amilly \\ Institut national de la Recherche agronomique
}

SOMMAIRE

Dans la souche dite "Bresse-pile " apparaissent régulièrement quelques animaux dont la crête et les barbillons présentent une coloration rose pâle au lieu de rouge vif. Les ségrégations observées montrent qu'on est en présence d'un caractère héréditaire simple, conditionné par un seul gène récessif autosomal. Il est probable qu'il s'agit de la même mutation que celle décrite par Deakin et RoBErtson sous le nom de "tête jaune " ou "tête pâle ".

\section{DESCRIPTION DU CARACTËEE}

La crête et les barbillons d'une poule domestique en bonne santé ont habituellement une couleur rouge vif, aussi bien chez les jeunes que chez les adultes. Mais dans la souche "Bresse-pile », qui est utilisée à la Station du Magneraud dans différents programmes expérimentaux, apparaissent régulièrement des animaux dont la crête et les barbillons présentent une coloration rose très pâle. Pour simplifier, nous avons donné à ce caractère le nom de "crête pâle ".

Il est probable que la couleur observée résulte d'une modification de la vascularisation des tissus qui forment la crête et les barbillons. En outre, la texture de ces organes est plus fine et leur épiderme plus doux au toucher. La distinction entre crête pâle et crête normale est facile à faire chez la poule, particulièrement à l'entrée en ponte. Chez la jeune poulette, le caractère s'observe d'autant mieux que la crête est plus développée. Chez le mâle, la distinction est facile à faire chez le jeune âgé de 2 à 3 mois. Vers l'âge de 5 mois, on observe encore une crête plus 
brillante et une texture plus fine, mais la couleur se rapproche de celle d'une crête normale. Par la suite, les deux types deviennent pratiquement indiscernables.

La souche Bresse-pile a été obtenue par Cochez à partir d'un croisement Bresse-blanche $\times$ New-Hampshire réalisé en I955. Presque tous les animaux ont la peau et les pattes blanches (gène $W$ ). Tous ont une crête simple, relativement développée, analogue à celle de la race Bresse. Le caractère "crête pâle " avait été observé à plusieurs reprises dans la souche Bresse utilisée (CochEz, communications personnelles). I1 a également été observé dans un autre troupeau de Bresse à la Station de Recherches avicoles de Jouy-en-Josas (MÉRAT, communications personnelles). Dans une souche de Plymouth-Rock barré, DEA KIN et ROBERTSON (I935) avaient décrit un caractère qu'ils avaient appelé " tête jaune " et qui semble correspondre au même phénomène que nous observons nous-mêmes, en particulier en ce qui concerne l'évolution de la coloration avec l'âge chez le mâle.

Nous présentons ici une étude génétique du caractère "crête pâle " faite à partir de ségrégations obtenues de I964 à I967 à la Station du Magneraud.

\section{ÉTUDE EXPÉRIMENTALE}

La souche Bresse-pile est reproduite chaque année à partir de 20 parquets pédigrés de 1 coq et 5 à 10 poules. Le caractère "crête pâle * est noté systématiquement depuis 1963. En 1963, les observations ont été faites à l'âge de 11 semaines. A partir de 1964, elles ont été faites à 8 , 11 et 20 semaines, âges qui correspondent à des manipulations habituelles pour les animaux de la souche.

Les crêtes ont été classées selon 3 phénotypes : pâle, normal ou douteux. Pour chaque poulet, nous avons considéré 2 observations : celle faite sur le jeune animal (phénotype à 8 semaines, confirmé ou non à 11 semaines) et celle faite sur l'animal âgé de 20 semaines. Nous n'avons conservé que les animaux qui avaient ces 2 observations. Pour tenir compte des différences de manifestation du caractère selon le sexe, nous avons adopté la grille de classification suivante :

\begin{tabular}{c|c|c|c}
\hline \hline $\begin{array}{c}\text { Notation } \\
\text { 8-i I semaines }\end{array}$ & $\begin{array}{c}\text { Notation } \\
\text { 20 semaines }\end{array}$ & Mâles & Femelles \\
\cline { 2 - 3 } $\begin{array}{c}\text { pâle } \\
\text { pâle } \\
\text { pâle } \\
\text { douteux } \\
\text { douteux } \\
\text { douteux } \\
\text { normal } \\
\text { normal } \\
\text { normal }\end{array}$ & $\begin{array}{c}\text { pâle } \\
\text { douteux } \\
\text { normal } \\
\text { pâle } \\
\text { douteux } \\
\text { normal } \\
\text { pâle } \\
\text { douteux } \\
\text { normal }\end{array}$ & $\begin{array}{c}\text { pâle } \\
\text { pâle } \\
\text { pâle } \\
\text { pâle } \\
\text { douteux } \\
\text { normal } \\
\text { normal } \\
\text { normal } \\
\text { normal }\end{array}$ & $\begin{array}{c}\text { pâle } \\
\text { pâle } \\
\text { normal } \\
\text { pâle } \\
\text { douteux } \\
\text { normal } \\
\text { pâle } \\
\text { normal } \\
\text { normal }\end{array}$ \\
\hline \hline
\end{tabular}

Nous avons étudié la descendance des couples où les 2 parents présentaient le caractère " crête pâle " ainsi que les cas où l'un des parents était " pâle " et l'autre " normal ", ce dernier étant lui-même issu d'un animal " pâle "et d'un animal " normal ". 


\section{RÉSULTATS ET DISCUSSION}

Le tableau I résume les résultats des accouplements coq " pâle " $\times$ poule " pâle ". La très grande majorité des enfants (265 sur 278) ressemblent aux parents. Quatre n'ont pu être classés de façon certaine et 9 présentent un phénotype normal. Parmi ces derniers, 3 étaient des poules notées "pâle " à II semaines et " normal " adulte; I coq a été noté " douteux " à II semaines et " normal " à 20 semaines, 2 coqs ont été notés " normal " à II semaines et "pâle " à 20 semaines, I coq et 2 poules présentaient un phénotype "normal " à la fois à II semaines et à 20 semaines. Il $\mathrm{y}$ avait donc 6 poulets plus ou moins difficiles à classer et 3 seulement dont le phénotype peut être considéré comme réellement normal.

TABLEAU I

Résultats des accouplements entre un coq "pâle " et une poule "pâle "

\begin{tabular}{|c|c|c|c|c|c|}
\hline \multirow{2}{*}{ Catégories } & \multirow{2}{*}{$\begin{array}{l}\text { Nombre } \\
\text { de couples } \\
\text { étudiés }\end{array}$} & \multicolumn{3}{|c|}{ Phénotype des enfants } & \multirow{2}{*}{ Total } \\
\hline & & Pâle & Normal & Douteux & \\
\hline $\begin{array}{l}\text { Mâles } \\
\text { Femelles. } \\
\text { Ensemble : mâles }+ \text { fe- } \\
\text { melles. }\end{array}$ & $\begin{array}{l}31 \\
36 \\
36\end{array}$ & $\begin{array}{r}125 \\
140 \\
265\end{array}$ & $\begin{array}{l}4 \\
5\end{array}$ & $\begin{array}{l}4 \\
0 \\
4\end{array}$ & $\begin{array}{l}133 \\
\text { I } 45 \\
278\end{array}$ \\
\hline
\end{tabular}

Étant donné le petit nombre d'enfants " non pâle " obtenus quand on croise 2 animaux à crête pâle, nous pouvons considérer que nous avons affaire à un caractère héréditaire simple. Les difficultés de classement peuvent conduire à des erreurs. Elles traduisent une expressivité du caractère qui peut varier d'un animal à l'autre. C'est ce qui peut expliquer l'apparition des poulets non pâles indiqués dans le tableau $x$.

Les modalités d'apparition du caractère " crête pâle " font penser à un déterminisme génétique de type récessif. L'hypothèse la plus simple est celle d'un seul gène récessif autosomal. Si cette hypothèse est vraie, les animaux à crête normale, fils d'un parent " normal " et d'un parent " pâle ", sont hétérozygotes. En croisement avec des animaux à crête pâle, on doit obteriir des enfants "normaux " et "pâles " selon la proportion I : I. C'est ce que vérifient les résultats consignés dans le tableau 2, aussi bien pour chaque type d'accouplement que pour l'ensemble des résultats. En particulier, la moitié des filles de coqs à crête pâle ont un phénotype normal, ce qui exclut 1'hypothèse d'un gène lié au sexe.

Le caractère " tête jaune " décrit par DEAKIN et ROBERTSON (I935) correspond à une modification de la vascularisation amenant une décoloration de la crête, des barbillons, des oreillons et même de la peau de la face. Chez des animaux à peau et pattes jaunes (correspondant au génotype $w$ ), toute la tête paraît 
TABLEAU 2

Résultats des accouplements entre un parent à crête pâle et un parent à crête normale mais supposé hétérozygote

\begin{tabular}{|c|c|c|c|c|c|}
\hline \multirow{2}{*}{ Catégories } & \multirow{2}{*}{$\begin{array}{c}\text { Nombre } \\
\text { de couples } \\
\text { étudiés }\end{array}$} & \multicolumn{3}{|c|}{ Phénotypes des enfants } & \multirow{2}{*}{ Total } \\
\hline & & Pâle & Normal & Douteux & \\
\hline
\end{tabular}

I $^{\circ}$ Père pâle. Mère normale, fille d'un coq pâle et d'une poule normale

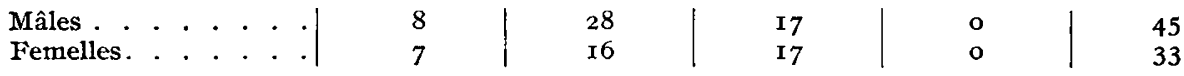

$2^{\circ}$ Père pâle. Mère normale, fille d'un coq normal et d'une poule pâle

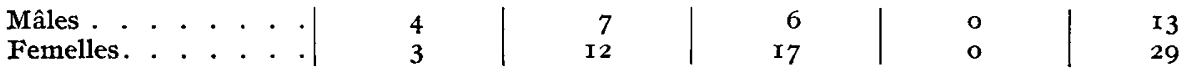

$3^{\circ}$ Mère pâle. Père normal, fils d'un coq pâle et d'une poule normale

\begin{tabular}{ll|l|l|l|l|r|r|} 
Mâles . . . . . . . . . . & . & 2 & 3 & 8 & 0 & II \\
Femelles. . . . . . . . & 2 & 4 & 4 & 0 & 8
\end{tabular}

$4^{\circ}$ Mère pâle. Père normal, fils d'un coq normal et d'une poule pâle

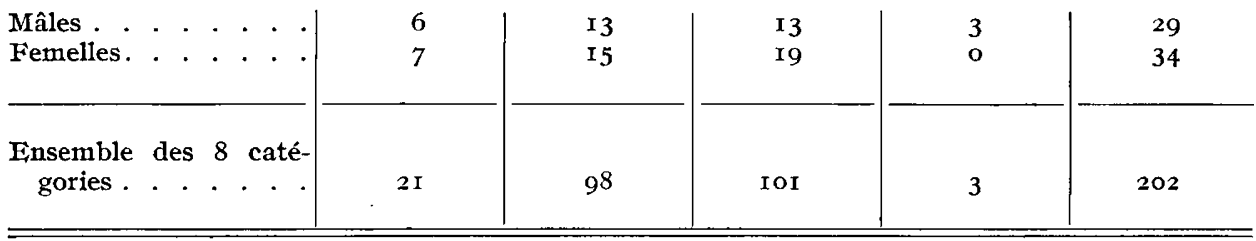

alors colorée en jaune. Les résultats de quelques accouplements ont permis à ces auteurs, d'émettre l'hypothèse d'un seul gène récessif autosomal, qu'ils ont appelé $g$, et qui est indépendant du gène $w$. Dans un travail ultérieur (DEAKIN et RoBERTSON, I937), ils ont fait ressortir la difficulté de bien discerner le caractère chez des animaux à peau et pattes blanches (gène W). Ils parlent alors de " tête pâle ". Cette observation confirme nos propres difficultés de classification, nos animaux étant aussi à peau et pattes blanches.

En définitive, les résultats que nous observons sont comparables à ceux de Deakin et Robertson. On peut penser qu'il s'agit de la même mutation apparue indépendamment dans 2 troupeaux différents.

Ręu pour publication en juillet r968.

\section{SUMMARY}

GENETIC CONTROL OF THE CHARACTER “ PALE COMB" IN THE DOMESTIC FOWL

In the so-called "Bresse-pile" strain have appeared regularly several animals whose comb and wattles were of a light pink color in place of bright red. A genetic study was carried out. Table 1 shows the results of matings where both parents had pale combs. Table 2 shows the results obtained when one of the parents had a pale comb while the other was normal, the latter 
being himself the progeny of one pale-comb parent and one normal-comb parent. The segregations obtained demonstrate that we have a simple hereditary character determined by a single autosomal recessive gene. It probably involves the same mutation as that described by DEA KIN and RoBERTSON entitled " yellow head" or " pale head".

\section{RÉFÉRENCES BIBLIOGRAPHIQUES}

DEAKIn A., Robertson G., 1935. The inheritance of yellow-pigmented heads in domestic fowl. Amer. Nat., 69, 378-380.

DEAkIN A., RoberTson G., 1937. I,inkage tests with the yellow-head and dominant-white plumage and white-skin characteristics in domestic fowl. Sci. Agric., 17, 45I-452. 